\title{
Average charge of superheavy recoil ion in helium gas
}

\author{
By D. Kaji ${ }^{1}$, , K. Morita ${ }^{1}$, K. Morimoto ${ }^{1}$, H. Haba ${ }^{1}$ and H. Kudo ${ }^{2}$ \\ ${ }^{1}$ Nishina Center for Accelerator Based Science, RIKEN, Wako, Saitama 351-0198, Japan \\ ${ }^{2}$ Department of Chemistry, Niigata University, Ikarashi, Niigata 950-2181, Japan
}

(Received November 27, 2009; accepted in final form Januar 25, 2011)

Superheavy element / Hot fusion / GARIS / Average charge / Trans-actinide / Recoil separator

Summary. The average equilibrium charges $q_{\text {ave }}$ of heavy recoil ions moving in helium gas were measured by a gasfilled recoil ion separator (GARIS). A new empirical formula to calculate $q_{\text {ave }}$ for superheavy recoil ions with a low velocity was derived. This formula was applicable to the search for a superheavy nuclide of ${ }^{266} \mathrm{Bh}$.

\section{Introduction}

A gas-filled recoil ion separator (GARIS) is one of the most active recoil separators in the search for a superheavy element (SHE). While operating a GARIS, the most important parameter is the average equilibrium charge $q_{\text {ave }}$ of a recoil ion moving in a filled gas because the trajectory of the recoil ion is governed by $B \rho=0.0227 \times A \times\left(v / v_{0}\right) / q_{\text {ave }}$, where $B \rho$ is the magnetic rigidity, $A$ is the mass number, and $v / v_{0}$ is the recoil velocity expressed in Bohr velocity units $\left(v_{0}=c / 137\right.$, where $c$ denotes the speed of light). In a previous study, we measured the $q_{\text {ave }}$ values of heavy recoil ions $\left({ }^{169} \mathrm{Tm},{ }^{208} \mathrm{~Pb},{ }^{193,209} \mathrm{Bi},{ }^{196} \mathrm{Po},{ }^{200} \mathrm{At},{ }^{203,204} \mathrm{Fr},{ }^{212} \mathrm{Ac}\right.$, ${ }^{234} \mathrm{Bk},{ }^{245} \mathrm{Fm},{ }^{254} \mathrm{No}$, and ${ }^{255} \mathrm{Lr}$ ) moving in helium gas using a GARIS [1] and, on the basis of the results, derived the following empirical formula for $q_{\text {ave }}$ in helium gas:

$$
q_{\text {ave }}=0.625 \times\left(v / v_{0}\right) \times Z^{1 / 3} .
$$

This formula is useful in the range $9.0 \leq\left(v / v_{0}\right) \times Z^{1 / 3} \leq$ 19.1. The formula was applied to the search for SHE nuclides of ${ }^{263,264,265} \mathrm{Hs},{ }^{271} \mathrm{Ds},{ }^{272} \mathrm{Rg},{ }^{277} 112$, and ${ }^{278} 113$ produced by cold fusion reactions [2-9].

In this work, the measurement of $q_{\text {ave }}$ was expanded for superheavy recoil ions produced by actinide-base fusion reactions.

\section{Experimental}

Heavy recoil ions were produced by actinide-based fusion reactions of ${ }^{238} \mathrm{U}\left({ }^{22} \mathrm{Ne}, 5 n\right){ }^{255} \mathrm{No}[10],{ }^{248} \mathrm{Cm}\left({ }^{18} \mathrm{O}, 5 n\right){ }^{261} \mathrm{Rf}$ [11], and ${ }^{248} \mathrm{Cm}\left({ }^{22} \mathrm{Ne}, 5 n\right){ }^{265} \mathrm{Sg}$ [12]. The evaporation residues (ERs) were separated from the projectiles, target re-

\footnotetext{
*Author for correspondence (E-mail: daiya@ riken.jp).
}

coils, and light-charged particles by the GARIS and collected at the focal plane of the separator. The GARIS was filled with helium gas at a pressure of approximately $30 \mathrm{~Pa}$. The optimum magnetic field for maximum transmission was identified. The nuclide was identified by two detection systems: In one system, the implantation and subsequent decays of ER were measured by a $60 \times 60 \mathrm{~mm}^{2} 12$-strip silicon detector installed at the focal plane of the GARIS. In the other, the ER was guided into a gas-jet chamber through a Mylar window, stopped in the helium gas, attached to $\mathrm{KCl}$ aerosol particles, and continuously transported through a Teflon capillary to a rotating wheel apparatus called MANON for $\alpha$ spectrometry. Subsequent decays of the ER were finally measured using MANON. Details of the GARIS + Gas-jet + MANON system are given elsewhere [13].

\section{Results and discussion}

Each intensity distribution of ${ }^{255} \mathrm{No},{ }^{261} \mathrm{Rf}$, and ${ }^{265} \mathrm{Sg}$ measured by changing the magnetic field setup of the GARIS is shown in Fig. 1. For ${ }^{255} \mathrm{No},{ }^{261} \mathrm{Rf}$, and ${ }^{265} \mathrm{Sg}$, maximum trans-

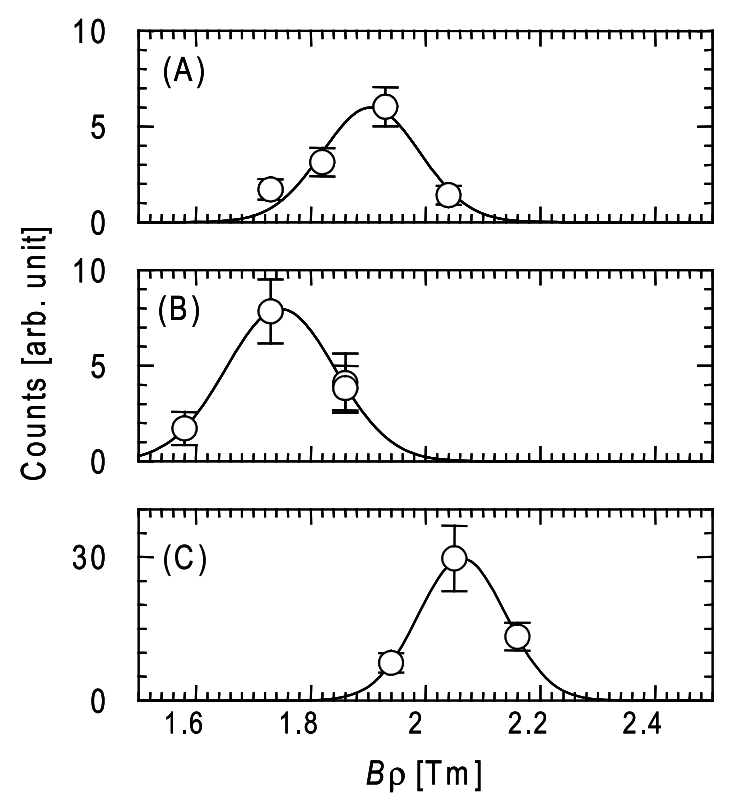

Fig. 1. Intensity distribution of (A) ${ }^{255} \mathrm{No}$, (B) ${ }^{261} \mathrm{Rf}$, and (C) ${ }^{265} \mathrm{Sg}$ ions measured by changing the magnetic field setup of the separator. Each solid curve is a fit to the data points with a Gaussian function. 

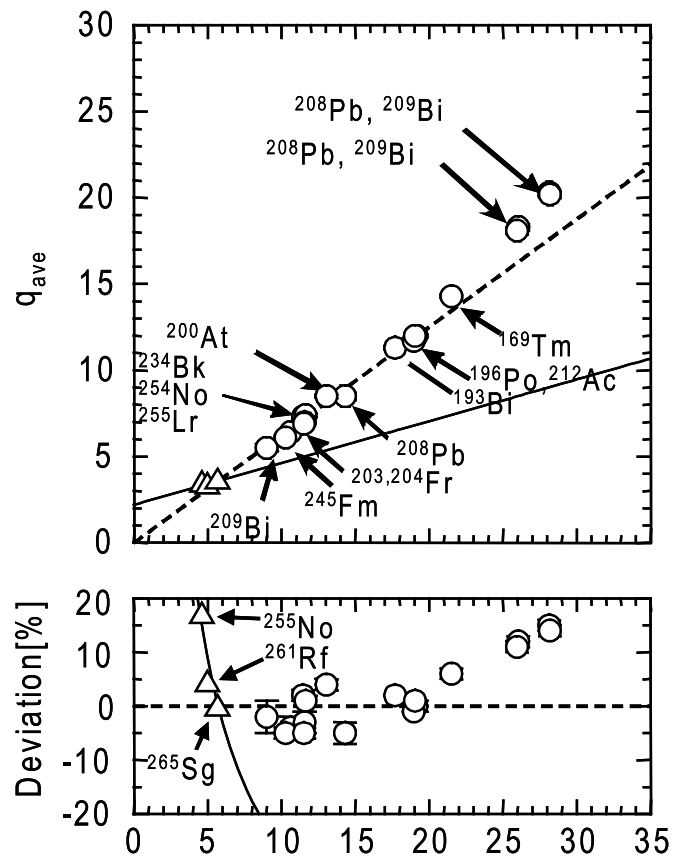

Fig. 2. [Upper part] The measured equilibrium charge $q_{\text {ave }}$ of heavy recoil ions moving in helium gas. Circles: previous work given in $[1,2]$ and squares: this work. Broken line: $q_{\text {ave }}=0.625 \times\left(v / v_{0}\right) \times Z^{1 / 3}$, solid line: $q_{\text {ave }}=0.242 \times\left(v / v_{0}\right) \times Z^{1 / 3}+2.19$. [Down part] Deviation from $q_{\text {ave }}$ values predicted by $q_{\text {ave }}=0.625 \times\left(v / v_{0}\right) \times Z^{1 / 3}$.

mission was obtained at $B \rho=1.91,1.75$, and 2.06 [Tm], and the widths $\Delta B \rho / B \rho=10.5,12.8$, and $8.7[\%]$. The $q_{\text {ave }}$ value was deduced from the $B \rho$ value corresponding the maximum collection of the ER. Each $q_{\text {ave }}$ is plotted against $\left(v / v_{0}\right) \times Z^{1 / 3}$ in Fig. 2. It was found that the deviation of $q_{\text {ave }}$ from our empirical formula in Eq. (1) increased with a decrease in $\left(v / v_{0}\right) \times Z^{1 / 3}$. Thus, a new empirical formula for the $q_{\text {ave }}$ value of recoils produced by actinide-based fusion reactions can be derived:

$$
q_{\text {ave }}=0.242 \times\left(v / v_{0}\right) \times Z^{1 / 3}+2.19,
$$

This formula is useful in the range $4.6 \leq\left(v / v_{0}\right) \times Z^{1 / 3} \leq 6.8$. A change in the slope was also observed in an early work using measurements of light recoil ions in helium gas [14]. The atomic shell structure of the recoil ion might influence the charge-exchanging process in helium gas. This new

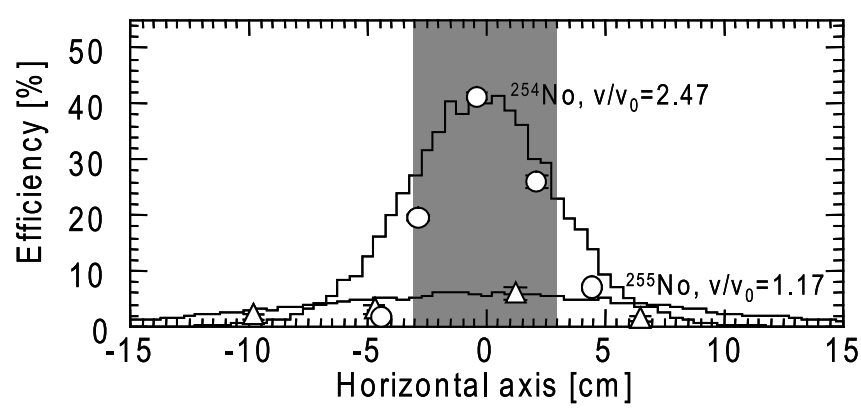

Fig. 3. Comparison of the image size between nobelium isotopes with different recoil velocities. Circles: ${ }^{254} \mathrm{No}$ given in [1,2], squares: ${ }^{255} \mathrm{No}$ in this work. Each simulation of the angular spread by multiple scattering of recoil ions with a filled gas is denoted by a solid line. An acceptance of the $60 \times 60 \mathrm{~mm}^{2} 12$-strips silicon detector is given in the hatched area.

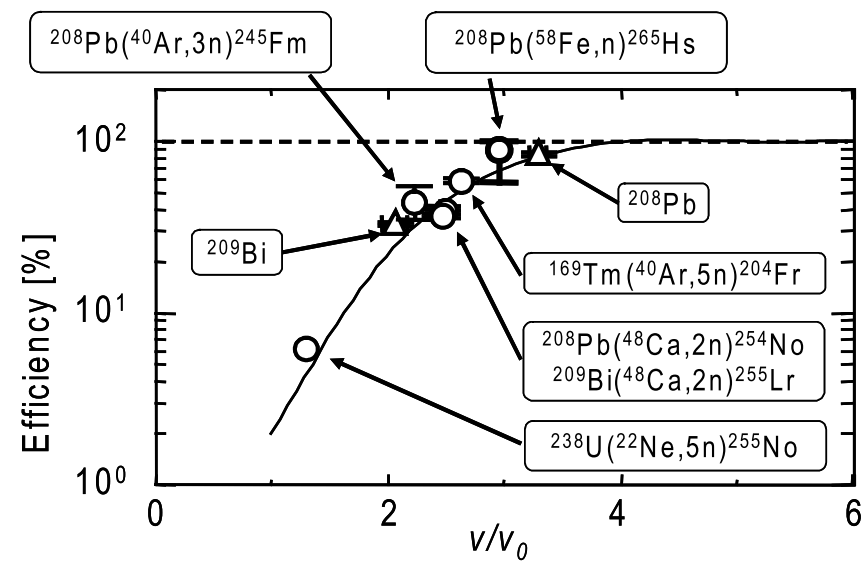

Fig. 4. Efficiency curve of the GARIS as a function of the recoil velocity expressed in Bohr velocity units.

formula was applied to search for a SHE nuclide of ${ }^{266} \mathrm{Bh}$ produced by a ${ }^{248} \mathrm{Cm}$-based fusion reaction [15].

The $\Delta B \rho / B \rho$ value increased with a decrease in the recoil velocity. This can be explained by the multiple scattering of the recoil ions by the helium gas atoms. For example, the difference in the image size at the focal plane of the separator between nobelium isotopes with different recoil velocities is shown in Fig. 3. Additionally, these positional distributions of the ions at the focal plane were also compared with a simulation assuming that the ions change their directions due to Rutherford scattering by the helium nuclei. This suggests that the transmission of the separator decreases at low recoil velocity. The efficiency curve of the GARIS is shown in Fig. 4.

Acknowledgment. This experiment was carried out at the RI Beam Factory operated by the RIKEN Nishina Center and CNS, University of Tokyo. These experiments were made possible because of the outstanding collaboration between accelerator scientists and technicians as well as nuclear physicists.

\section{References}

1. Kaji, D., Morita, K., Morimoto, K., Zhao, Y.-L., Yoneda, A., Suda, T., Yoshida, A., Kudo, H., Katori, K., Tanihata, I.: Measurement of equilibrium charge state of heavy ions in helium gas using a RIKEN gas-filled recoil separator. RIKEN Accel. Prog. Rep. 36, 89(2002).

2. Kaji, D.: Equilibrium charge of heavy recoil atom in helium gas, the way to a new element discovery. Doctoral dissertation, Niigata University (2003).

3. Kaji, D., Morimoto, K., Sato, N., Ichikawa, T., Ideguchi, E., Ozeki, K., Haba, H., Koura, H., Kudou, Y., Ozawa, A., Yoneda, A., Yohsida, A., and Morita, K.: Production and decay properties of ${ }^{263}$ Hs. J. Phys. Soc. Jpn. 78, 035003 (2009).

4. Sato, N.: Study on production and decay properties of neutron deficient Hs isotopes produced by cold fusion reactions. Doctoral dissertation, Tohoku University (2009).

5. Morita, K., Morimoto, K., Kaji, D., Haba, H., Ideguchi, E., Kanungo, R., Katori, K., Koura, H., Kudo, H., Ohnishi, T., Ozawa, A., Suda, T., Sueki, K., Tanihata, I., Xu, H., Yeremin, A. V., Yoneda, A., Yoshida, A., Zhao, Y.-L., Zheng, T.: Production and decay of the isotope ${ }^{271}$ Ds $(Z=110)$. Eur. Phys. J. A 21, 257 (2004).

6. Morita, K., Morimoto, K., Kaji, D., Haba, H., Ideguchi, E., Peter, J. C., Kanungo, R., Katori, K., Koura, H., Kudo, H., Ohnishi, T., Ozawa, A., Suda, T., Sueki, K., Tanihata, I., Xu, H., Yeremin, A. V., Yoneda, A., Yoshida, A., Zhao, Y.-L., Zheng, T., 
Goto, S., and Tokanai, F.: Production and decay properties of ${ }^{272} 111$ and its daughter nuclei. J. Phys. Soc. Jpn. 73, 1738 (2004).

7. Morita, K., Morimoto, K., Kaji, D., Akiyama, T., Goto, S., Haba, H., Ideguchi, E., Katori, K., Koura, H., Kudo, H., Ohnishi, T., Ozawa, A., Suda, T., Sueki, K., Yamaguchi, T., Yoneda, A., and Yoshida, A.: Experiment on the synthesis of an isotope ${ }^{277} 112$ by ${ }^{208} \mathrm{~Pb}\left({ }^{70} \mathrm{Zn}, n\right)^{277} 112$. J. Phys. Soc. Jpn. 76, 043201 (2007).

8. Morita, K., Morimoto, K., Kaji, D., Akiyama, T., Goto, S., Haba, H., Ideguchi, E., Kanungo, R., Katori, K., Koura, H., Kudo, H., Ohnishi, T., Ozawa, A., Suda, T., Sueki, K., Xu, H., Yamaguchi, T., Yoneda, A., Yoshida, A., and Zhao, Y.-L.: Experiment on the synthesis of element 113 in the reaction ${ }^{209} \mathrm{Bi}\left({ }^{70} \mathrm{Zn}, n\right)^{278} 113$. J. Phys. Soc. Jpn. 73, 2593 (2004)

9. Morita, K., Morimoto, K., Kaji, D., Akiyama, T., Goto, S., Haba, H., Ideguchi, E., Katori, K., Koura, H., Kikunaga, H., Kudo, H., Ohnishi, T., Ozawa, A., Sato, N., Suda, T., Sueki, K., Yamaguchi, T., Yoneda, A., and Yoshida, A.: Observation of second decay chain from ${ }^{278} 113$. J. Phys. Soc. Jpn. 76, 045001 (2007).

10. Haba, H., Kikunaga, H., Kaji, D., Akiyama, T., Morimoto, K., Morita, K., Nanri, T., Ooe, K., Sato, N., Shinohara, A., Suzuki, D., Takabe, T., Yamazaki, I., Yokoyama, A., and Yoneda, A.: Performance of the gas-jet transport system coupled to the RIKEN gas-filled recoil ion separator GARIS for the ${ }^{238} \mathrm{U}\left({ }^{22} \mathrm{Ne}, 5 n\right)^{255} \mathrm{No}$ reaction. J. Nucl. Radiochem. Sci. 9, 27 (2008).
11. Haba, H., Kaji, D., Komori, Y., Kudou, Y., Morimoto, K., Morita, K., Ooe, K., Ozeki, K., Sato, N., Shinohara, A., and Yoneda, A.: RIKEN gas-filled recoil ion separator (GARIS) as a promising interface for superheavy element chemistry - production of element $104,{ }^{261} \mathrm{Rf}$, using the GARIS/Gas-jet system -. Chem. Lett. 38 , 426 (2009).

12. Haba, H.: RIKEN GARIS for superheavy element chemistry. $7^{\text {th }}$ Workshop on Recoil Separator for Superheavy Element Chemistry (TASCA08), 31 October 2008, Darmstadt, Germany.

13. Haba, H., Kaji, D., Kikunaga, H., Akiyama, T., Sato, N., Morimoto, K., Yoneda, A., Morita, K., Takabe, T., and Shinoharad, A.: Development of gas-jet transport system coupled to the RIKEN gas-filled recoil ion separator GARIS for superheavy element chemistry. J. Nucl. Radiochem. Sci. 8, 55(2007).

14. Oganessian, Yu. Ts., Lobanov, Yu. V., Popeko, A. G., Abdullin, F. Sh., Kharitonov, Yu. P., Ledovskoy, A. A., and Tsyganov, Yu. S.: The average equilibrium charge states of heavy ions with $Z>60$ stripped in $\mathrm{He}$ and $\mathrm{H}_{2}$. Z. Phys D21, S357 (1991).

15. Morita, K., Morimoto, K., Kaji, D., Haba, H., Ozeki, K., Kudou, Y., Sato, N., Sumita, T., Yoneda, A., Ichikawa, T., Fujimori, Y., Goto, S., Ideguchi, E., Kasamatsu, Y., Katori, K., Komori, Y., Koura, H., Kudo, H., Ooe, K., Ozawa, A., Tokanai, F., Tsukada, K., Yamaguchi, T., and Yoshida, A.: Decay properties of ${ }^{266} \mathrm{Bh}$ and ${ }^{262} \mathrm{Db}$ produced in the ${ }^{248} \mathrm{Cm}+{ }^{23} \mathrm{Na}$ reaction. J. Phys. Soc. Jpn. 78, 064201(2009). 